\title{
Thermal noise can facilitate energy conversion by a ratchet system
}

\author{
Fumiko Takagi* and Tsuyoshi Hondou ${ }^{\dagger}$ \\ Department of Physics, Tohoku University \\ Sendai 980-8578, Japan
}

(Received 26 March 1999)

\begin{abstract}
Molecular motors in biological systems are expected to use ambient fluctuation. In a recent Letter [Phys. Rev. Lett. 80, 5251 (1998)], it was showed that the following question was unsolved, "Can thermal noise facilitate energy conversion by ratchet system?" We consider it using stochastic energetics, and show that there exist systems where thermal noise helps the energy conversion.

05.40.-a, 87.10.+e
\end{abstract}

Typeset using REVTEX

*Electronic address: fumiko@cmpt01.phys.tohoku.ac.jp

†Electronic address: hondou@cmpt01.phys.tohoku.ac.jp 
Molecular motors in biological systems are known to operate efficiently [1] They convert molecular scale chemical energy into macroscopic mechanical work with high efficiency in water at room temperature, where the effect of thermal fluctuation is unavoidable. These experimental facts lead us to expect the existence of the system where thermal noise helps the operation. To find out the mechanism of these motors is interesting not only to biology but also to statistical and thermal physics.

Recently inspired by observations on the molecular motors, many studies have been performed from the viewpoint of statistical physics. Much has been studied in ratchet models [5 [7] to consider how the directed motion appears from non-equilibrium fluctuation. One of the best known works among these ratchet models was by Magnasco $[\mathbb{8}$. He studied "forced thermal ratchet," and claimed that "there is a region of the operating regime where the efficiency is optimized at finite temperatures." His claim is interesting because thermal noise is usually known to disturb the operation of machines. However, recently it was revealed that this claim was made incorrectly [9], because it was not based on the analysis of the energetic efficiency but only on that of the probability current, as most of the studies of ratchet systems were. The insufficient analysis was attributed to the lack of systematic method of energetics in systems described by Langevin equation. Recently a method what is called stochastic energetics was formalized, where the heat was described quantitatively in the frame of Langevin equation [10]. Using this method, some attempts to discuss the energetics of these systems [11 [14] have been made. By the energetic formulation of the forced thermal ratchet [9] using this stochastic energetics, the following was showed: The behavior of the probability current is qualitatively different than that of energetic efficiency. Thermal noise does not help the energy conversion by the ratchet at least on the condition where the claim was made.

Therefore it was revealed that the following question had not yet been solved, "Can thermal noise facilitate operation of the ratchet?" In this Letter, we will show that the thermal noise certainly can facilitate the operation of the ratchet.

Let us consider an over-dumped particle in an "oscillating ratchet", where the amplitude 
of the 1-D ratchet potential is constant, but the degree of the symmetry breaking oscillates at frequency $\omega$ (Fig. 11). Langevin equation is as follows:

$$
\begin{aligned}
\frac{d x}{d t} & =-\frac{\partial V(x, t)}{\partial x}+\xi(t), \\
V(x, t) & =V_{p}(x, t)+\ell x,
\end{aligned}
$$

where $x, \ell$ and $V_{p}(x, t)$ represent the state of the system, the load and the ratchet potential respectively (Fig. 2) . The white and Gaussian random force $\xi(t)$ satisfies $\langle\xi(t)\rangle=0$ and $\left\langle\xi(t) \xi\left(t^{\prime}\right)\right\rangle=2 \epsilon \delta\left(t-t^{\prime}\right)$, where the angular bracket $\langle\cdot\rangle$ denotes the ensemble average. We use the unit $m=\gamma=1$. We assume that the potential $V(x, t)$ always has basins, and thus a particle cannot move over the potential peak without thermal noise. The ratchet $V_{p}(x, t)$ is assumed to satisfy the temporally and spatially periodic conditions,

$$
\begin{aligned}
& V_{p}(x, t+T)=V_{p}(x, t), \\
& V_{p}(x+L, t)=V_{p}(x, t),
\end{aligned}
$$

where $L$ is a spatial period of the ratchet potential, and $T\left(\equiv \frac{2 \pi}{\omega}\right)$ is a temporal period of the potential modulation. By potential modulation, energy is introduced into the system, and the system converts it into work against the load [15].

The Fokker-Planck equation [16] corresponding to Eq. (11) is written

$$
\begin{aligned}
\frac{\partial P(x, t)}{\partial t} & =-\frac{\partial J(x, t)}{\partial x} \\
& =-\frac{\partial}{\partial x}\left(-\frac{\partial V(x, t)}{\partial x} P(x, t)\right)+\epsilon \frac{\partial^{2} P(x, t)}{\partial x^{2}}
\end{aligned}
$$

where $P(x, t)$ and $J(x, t)$ are a probability density and a probability current respectively. We apply the periodic boundary conditions on $P(x, t)$ and $J(x, t)$,

$$
\begin{aligned}
& P(x+L, t)=P(x, t), \\
& J(x+L, t)=J(x, t),
\end{aligned}
$$

where $P(x, t)$ is normalized in the spatial period $L$. Except for transient time, $P(x, t)$ and $J(x, t)$ satisfy the temporally periodic conditions 


$$
\begin{gathered}
P(x, t+T)=P(x, t), \\
J(x, t+T)=J(x, t) .
\end{gathered}
$$

According to the stochastic energetics [10], the heat $\widetilde{Q}$ released to the heat bath during the period $T$ is given as,

$$
\widetilde{Q}=\int_{x(0)}^{x(T)}\left\{-\left(-\frac{d x(t)}{d t}+\xi(t)\right)\right\} d x(t) .
$$

Inserting Eq. (1) into Eq. (10), we obtain the energy balance equation,

$$
\widetilde{Q}=\int_{0}^{T} \frac{\partial V(x(t), t)}{\partial t} d t-\int_{V(0)}^{V(T)} d V(x(t), t) .
$$

The first term of RHS is the energy $\widetilde{E_{i n}}$ that the system obtain through the potential modulation, and the second term, $\int_{V(0)}^{V(T)} d V(x(t), t)$, is the work $\widetilde{W}$ that the system extracts from the input energy $\widetilde{E_{\text {in }}}$, during the period $T$. The ensemble average of $\widetilde{W}$ is given using Eqs. (2), (3) and (8) as,

$$
\begin{aligned}
\langle\widetilde{W}\rangle & =\left\langle\int_{V(0)}^{V(T)} d V(x(t), t)\right\rangle \\
& =\ell \int_{0}^{T} d t \int_{0}^{L} d x J(x, t) \equiv W,
\end{aligned}
$$

where one can find that $W$ represents the work against the load. Also, using Eqs. (2), (5) and the periodic conditions (Eqs. (3), (4), (7) and (8)), the ensemble average of $E_{\text {in }}$ is given as

$$
\begin{aligned}
\left\langle\widetilde{E_{\text {in }}}\right\rangle & =\left\langle\int_{0}^{T} \frac{\partial V(x(t), t)}{\partial t} d t\right\rangle \\
& =\int_{0}^{T} d t \int_{0}^{L} d x\left(-\frac{\partial V_{p}(x, t)}{\partial x}\right) J(x, t) \equiv E_{i n} .
\end{aligned}
$$

Taking an ensemble average, Eq. (11) yields,

$$
\begin{aligned}
Q= & E_{i n}-W \\
= & \int_{0}^{T} d t \int_{0}^{L} d x\left(-\frac{\partial V_{p}(x, t)}{\partial x}\right) J(x, t) \\
& \quad-\ell \int_{0}^{T} d t \int_{0}^{L} d x J(x, t),
\end{aligned}
$$


where $Q \equiv\langle\widetilde{Q}\rangle$. Therefore we obtain the efficiency $\eta$ of the energy conversion from the input energy $E_{\text {in }}$ into the work $W$, as follows,

$$
\eta=\frac{W}{E_{\text {in }}}=\frac{\ell \int_{0}^{T} d t \int_{0}^{L} d x J(x, t)}{\int_{0}^{T} d t \int_{0}^{L} d x\left(-\frac{\partial V_{p}(x, t)}{\partial x}\right) J(x, t)} .
$$

This expression can be estimated simply by solving the Fokker-Planck equation (Eq. (5)).

We solve Eq. (5) numerically with the following ratchet potential as an example. It satisfies Eqs. (3), (四) and the condition that the degree of the asymmetry oscillates but the amplitude of the ratchet is constant. It will turn out that the results does not depend on the detailed shape of the potential. The ratchet potential is

$$
V_{p}(x, t)=\frac{1}{2} V_{0}\left(\sin \left(\frac{2 \pi x}{L}+A(t) \sin \left(\frac{2 \pi x}{L}+C_{1} \sin \left(\frac{2 \pi x}{L}\right)\right)\right)+1\right)
$$

where $A(t)=C_{2}+C_{3} \sin (\omega t)$, and $V_{0}, C_{1}, C_{2}, C_{3}$ are constant.

The results are shown in Fig. 3. We find that the efficiency is maximized at finite intensity of thermal noise (Fig. 3(a)). This shows that thermal noise can certainly facilitate the energy conversion. What is the reason for the behavior of the efficiency $\eta$ ? Let us see the work $W$ and the input energy $E_{i n}$ as a function of the intensity of thermal noise. The work $W$, the numerator of Eq. (17), has a peak at finite intensity of thermal noise (Fig. 3(b)), because of the behavior of the flow during the period $T, \bar{J} \equiv \int_{0}^{T} d t \int_{0}^{L} d x J$. In the absence of thermal noise $(\epsilon=0)$, the particle cannot move over the potential peak (which results in $\bar{J}=0)$. As the intensity of thermal noise increases, the effect of non-equilibility emerges and it induces finite asymmetric flow against the load through the asymmetry of the ratchet. When thermal noise is large enough $(\epsilon \rightarrow \infty)$, the flow against load is no longer positive, because the effect of the ratchet disappears in this limit. Therefore the flow, and also the work, behave like Fig. 3(b) as a function of thermal noise intensity. The input energy $E_{\text {in }}$, the denominator of Eq. (17), remains finite at the limit $\epsilon \rightarrow 0$ (Fig. 3(c)), where all input energy dissipates because the oscillation of the local potential minimum makes finite local current even in the absence of thermal noise. Therefore the efficiency starts with $\eta=0$ at $\epsilon=0$, and grows up as the intensity of thermal noise increases, then disappears as $\epsilon \rightarrow \infty$. 
The efficiency has its peak at finite $\epsilon$.

As we have stated above, noise-induced flow and finite dissipation in the absence of thermal noise are the cause for the noise-induced energy conversion. Thus our finding will not depend on the detail of the shape of $V_{p}(x, t)$. We expect that thermal noise can facilitate the energy conversion in a variety of ratchet systems.

Finally we discuss the forced thermal ratchet [ 8$]$. The forced thermal ratchet is a system where a dissipative particle in a ratchet is subjected both to zero-mean external force and to thermal noise. The previous Letter [9] was the first trial that discussed the energetics in the ratchet. For the analytical estimate, the discussion in that Letter was only on the quasi-static limit where the change of the external force is slow enough. In that case, thermal noise cannot facilitate operation of the ratchet. The energetic efficiency is monotonically decreasing function of thermal noise intensity, in contrast to the oscillating ratchet discussed above. However one notices that the external force of the forced thermal ratchet can also be written by oscillatory modulating potential, when the external force is periodic as in the literature [8.9], It is likely that the difference between the two cases, the oscillating ratchet and the forced thermal ratchet discussed in that Letter [9], is attributed to the condition of the system, namely, quasi-static or not. We suppose that thermal noise may facilitate the energy conversion in the forced thermal ratchet when the ratchet is not quasi-static.

Langevin equation of the forced thermal ratchet is the same as Eq. (1), except for the potential $V$. In this case, the potential is

$$
V(x, t)=V_{p}(x)+\ell x-F_{e x}(t) x,
$$

where $V_{p}(x), \ell$ and $F_{e x}$ represent the ratchet potential, load and an external force respectively. The periodic external force $F_{e x}(t)$ satisfies $F_{e x}(t+T)=F_{e x}(t)$ and $\int_{0}^{T} d t F_{e x}(t)=0$ [17]. The work $W$ is the same as Eq. (13), and the input energy $E_{\text {in }}$ is,

$$
E_{\text {in }}=\int_{0}^{T} d t \int_{0}^{L} d x F_{e x}(t) J(x, t) .
$$

In quasi-static limit 9], the probability current $J$ does not depend on the coordinate $x$. Thus, when the current over the potential peak (that causes $W$ ) vanishes, the local current 
vanishes anywhere $(J(x, t)=J(t)=0)$. However, if the system is not quasi-static, the behavior changes qualitatively. In this case, even when the current over the potential peak vanishes at $\epsilon=0$, local current around the local potential minimum still remains finite. Thus there exists finite energy dissipation even in the limit $\epsilon \rightarrow 0$, which means that the input energy $E_{\text {in }}$ still remains finite value at this limit (Fig. \&(c)). Therefore, the efficiency is found to be zero at $\epsilon=0$, and has a peak at finite $\epsilon$ (Fig. 4(a)). The result is the same as that of the oscillating ratchet. It must be noted that the energetics can distinguish the behavior of the efficiency in the non-quasistatic case from that in quasi-static case, although the dependences of the flow $\bar{J}$ are the same between the two.

We have discussed energetics of the ratchet system using the method of the stochastic energetics, and estimated the efficiency of energy conversion. We found that thermal noise can facilitate the operation of the ratchet system. The mechanism was briefly summarized as follows: Through the ratchet, potential modulation causes noise-induced flow against the load that results in the work. On the other hand, potential modulation with finite speed causes local current around the local potential minimum that makes finite dissipation even in the absence of thermal noise. Thus the efficiency is maximized at finite intensity of thermal noise. The result must be robust and independent of the detail of the potential, because only two factors are essential for the energy conversion activated by thermal noise: One is the noise-induced flow, and the other is the finite dissipation in the absence of thermal noise. Also in the two-state model [7] that is an other type of ratchet systems, it was reported quite recently that the efficiency could be maximized at finite temperature [18]. We expect it to be examined by experiment whether and how the real molecular motors use thermal noise.

We would like to thank K. Sekimoto, J. Prost, A. Parmeggiani, F. Jülicher, S. Sasa, T. Fujieda and T. Tsuzuki for helpful comments. This work is supported by the Japanese Grant-in-Aid for Science Research Fund from the Ministry of Education, Science and Culture (No. 09740301) and Inoue Foundation for Science. 


\section{REFERENCES}

[1] T. Yanagida, T. Arata, and F. Oosawa, Nature 316, 366 (1985).

[2] A. Ishijima, H. Kojima, H. Higuchi, Y. Harada, T. Funatsu, and T. Yanagida, Biophys. J. 70, 383 (1996).

[3] T. Q. P. Uyeda, S. J. Kron, and J. A. Spudich, J. Mol. Biol. 214, 699 (1990).

[4] R. Yasuda, H. Noji, K. Kinosita, F. Motojima, and M. Yoshida, J. Bioenerg. Biomembr. 29, 207 (1997).

[5] R. D. Vale and F. Oosawa, Adv. Biophys., 26, 97 (1990).

[6] A. F. Huxley and R. M. Simmons, Nature, 233, 533 (1971).

[7] See, e.g., F. Jülicher, A. Ajdari and J. Prost, Rev. Mod. Phys. 691269 (1997), and references therein.

[8] M. O. Magnasco, Phys. Rev. Lett. 71, 1477 (1993).

[9] H. Kamegawa, T. Hondou and F. Takagi, Phys. Rev. Lett., 80, 5251 (1998).

[10] K. Sekimoto, J. Phys. Soc. Jpn. 66, 1234 (1997).

[11] K. Sekimoto and S. Sasa, J. Phys. Soc. Jpn., 66, 3326 (1997).

[12] M. Matsuo and S. Sasa, Physica A (to be published).

[13] T. Hondou and F. Takagi, J. Phys. Soc. Jpn., 67, 2974, (1998)

[14] K. Sekimoto, F. Takagi and T. Hondou, cond-mat/9904322.

[15] In this paper, we discuss the systems which convert mechanical energy into mechanical work, while real molecular motors in biological systems convert chemical energy into mechanical work. Recently an experiment suggests that the protein can store the chemical energy from ATP hydrolysis [19], the energy of which may be stored in mechanical 
way, for example, by conformational change of the protein. Some models have been proposed to explain this kind of energy storage [20].

[16] H. Risken, The Fokker-Planck Equation 2nd ed., (Springer-Verlag Berlin, 1989).

[17] We consider the low amplitude regime [9] where the amplitude of $F_{e x}(t)$ is small. In this case, a particle cannot move over the potential peak without thermal noise, as in case of the oscillating ratchet.

[18] A. Parmeggiani, F. Jülicher, A. Ajdari and J. Prost, cond-mat/9904153.

[19] A. Ishijima, H. Kojima, T. Funatsu, M. Tokunaga, H. Higuchi, H. Tanaka, and T. Yanagida, Cell 92, 161 (1998).

[20] For example, N. Nakagawa and K. Kaneko, chao-dyn/9903005.

[21] R. P. Feynman, R. B. Leighton and M. Sands, The Feynman Lectures in Physics (Addison-Wesley Publ. Co., Reading, Massachussets, 1966), vol. I. 


\section{FIGURES}

FIG. 1. Oscillating ratchet potential $V_{p}(x, t)$. The ratchet potential changes continuously between solid line and broken line with the time period $T$. The amplitude of the ratchet keeps constant, $V_{0}$.

FIG. 2. Snapshot of the potential $V(x, t)$ (solid line). Broken line represents the load term, $\ell x$.

FIG. 3. The energetic efficiency, $\eta=W / E_{i n}$, of the oscillating ratchet system as a function of thermal noise intensity, where $V_{0} / \omega L^{2}=0.01, \ell / \omega L=0.00002, C_{1}=0.3, C_{2}=0.3$ and $C_{3}=0.3$. (a) the efficiency $\eta$, (b) the work $W$ and (c) the input energy $E_{i n}$.

FIG. 4. The energetic efficiency, $\eta=W / E_{i n}$, of the forced thermal ratchet as a function of

thermal noise intensity, where $V_{0} / \omega L^{2}=1.0, \ell / \omega L=0.001$ and $\left|F_{e x}\right|_{\max } / \omega L=1.0$. (a) the efficiency $\eta$, (b) the work $W$ and (c) the input energy $E_{\text {in }}$. 


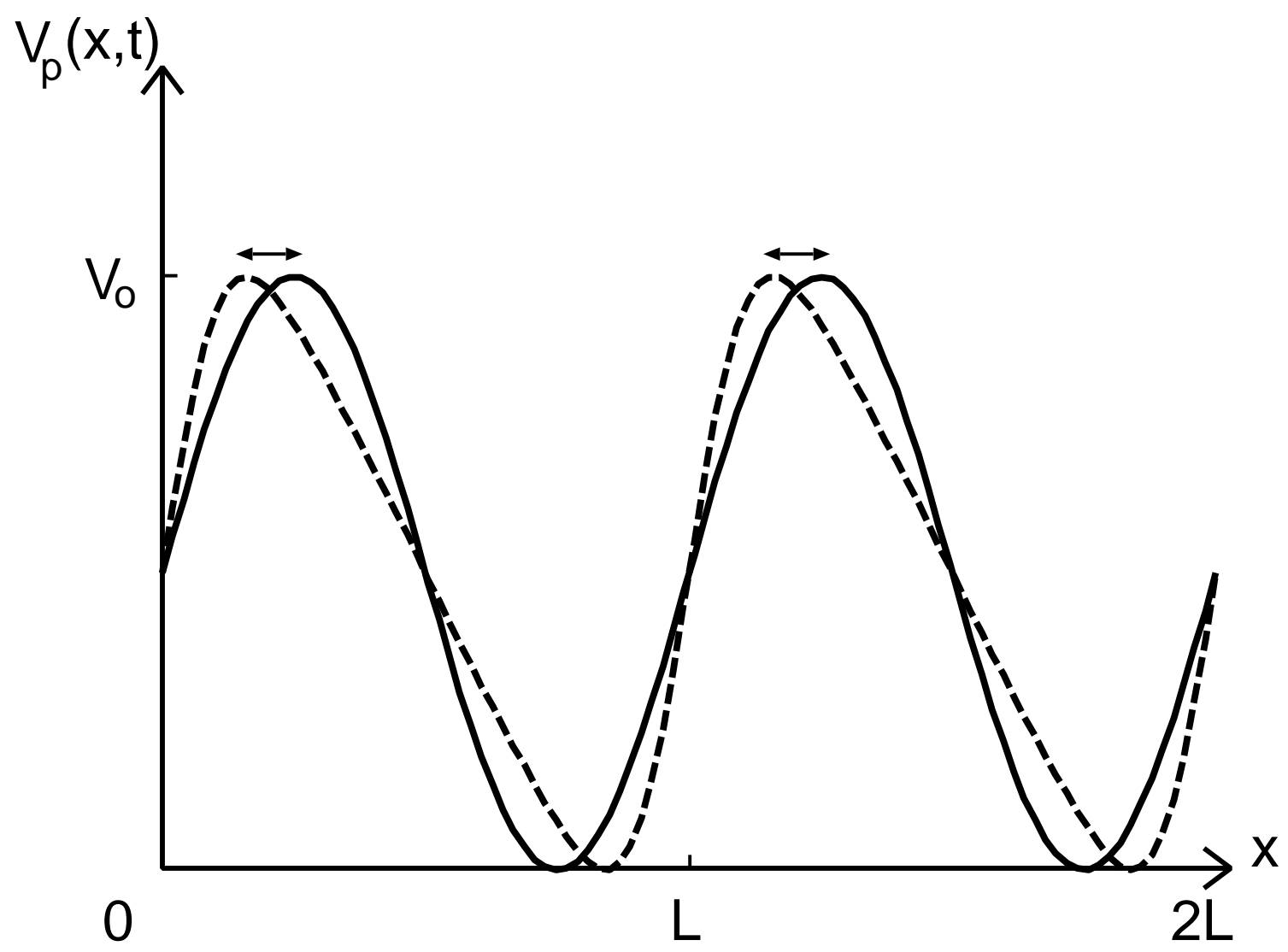




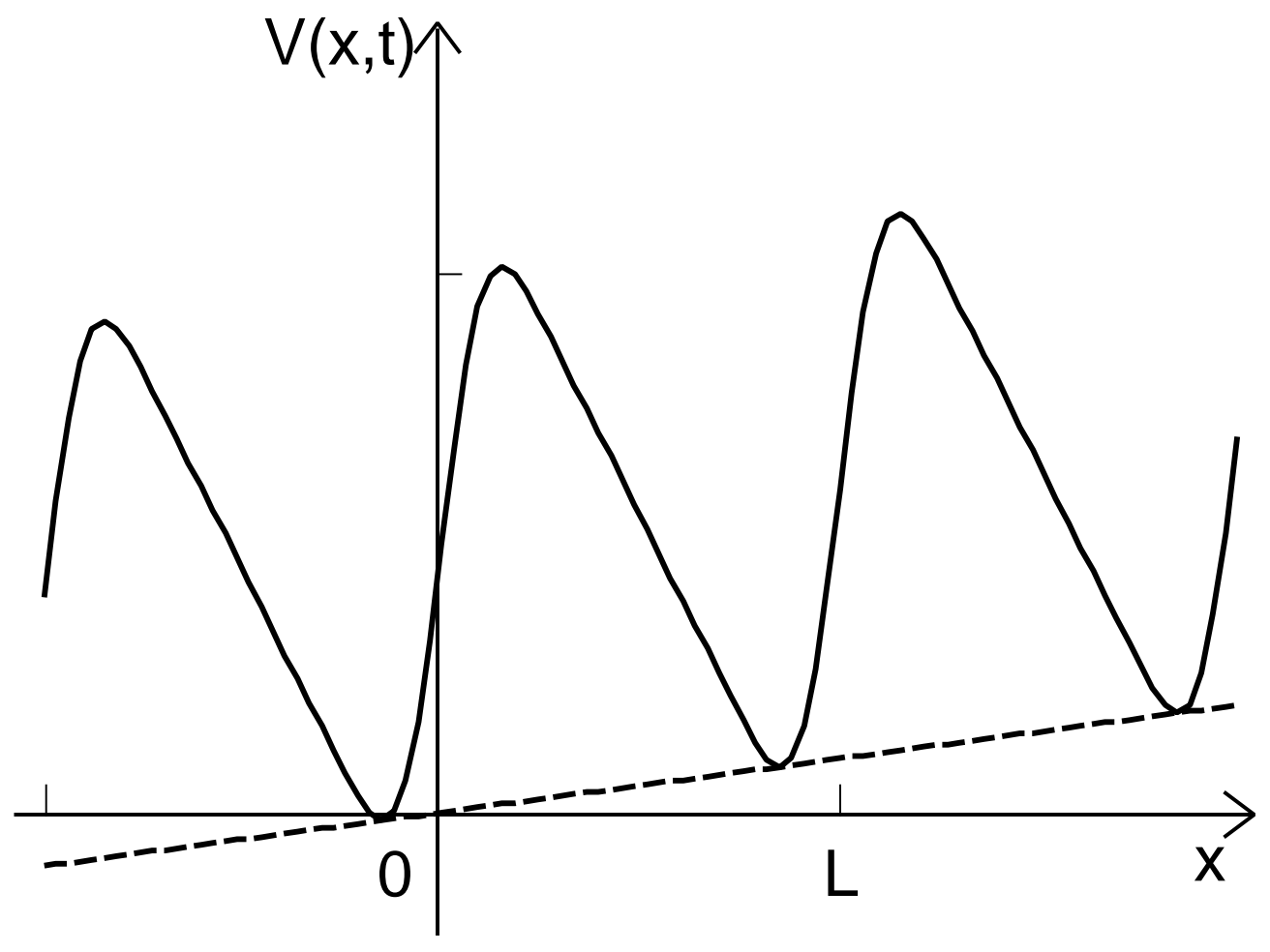




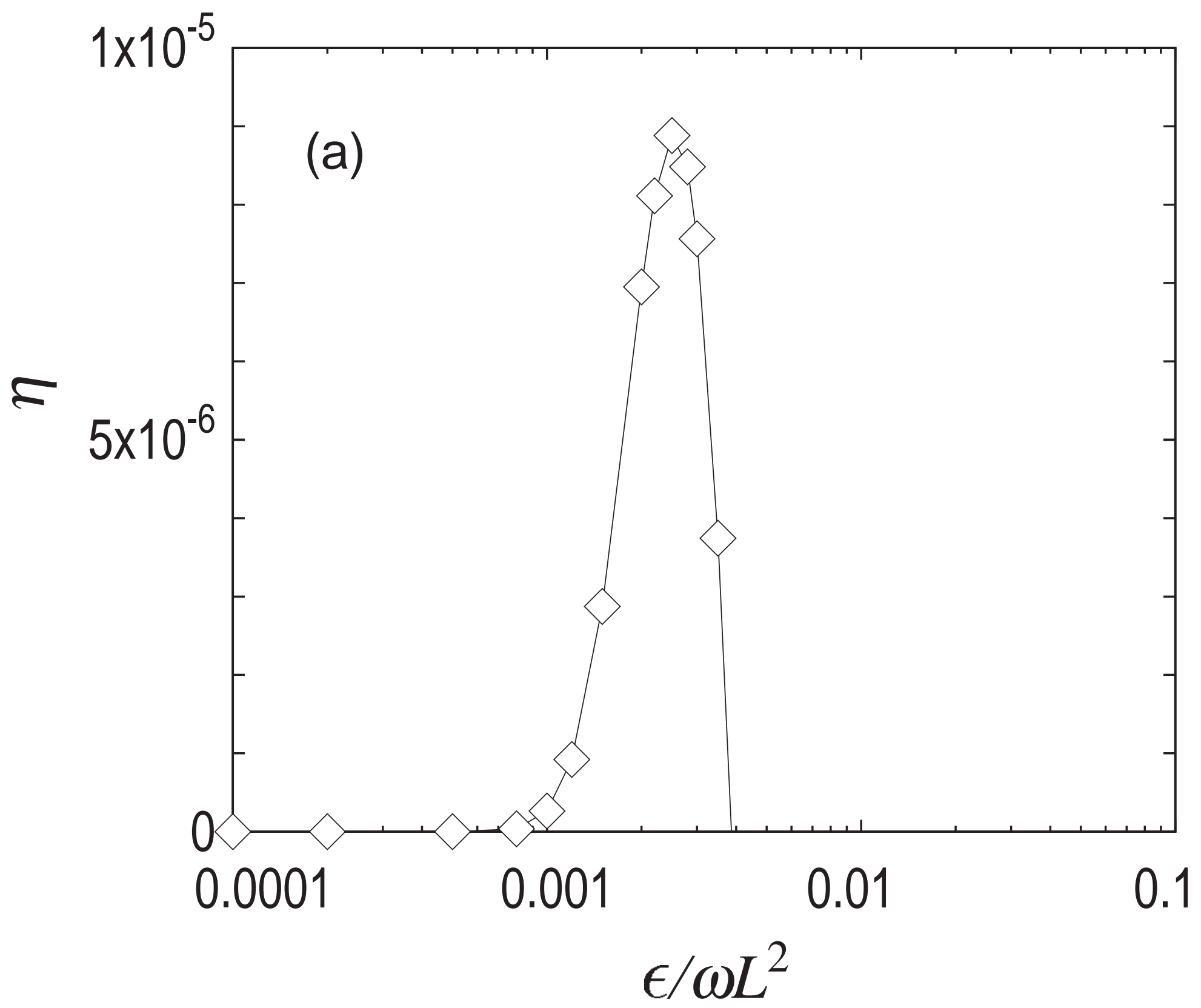




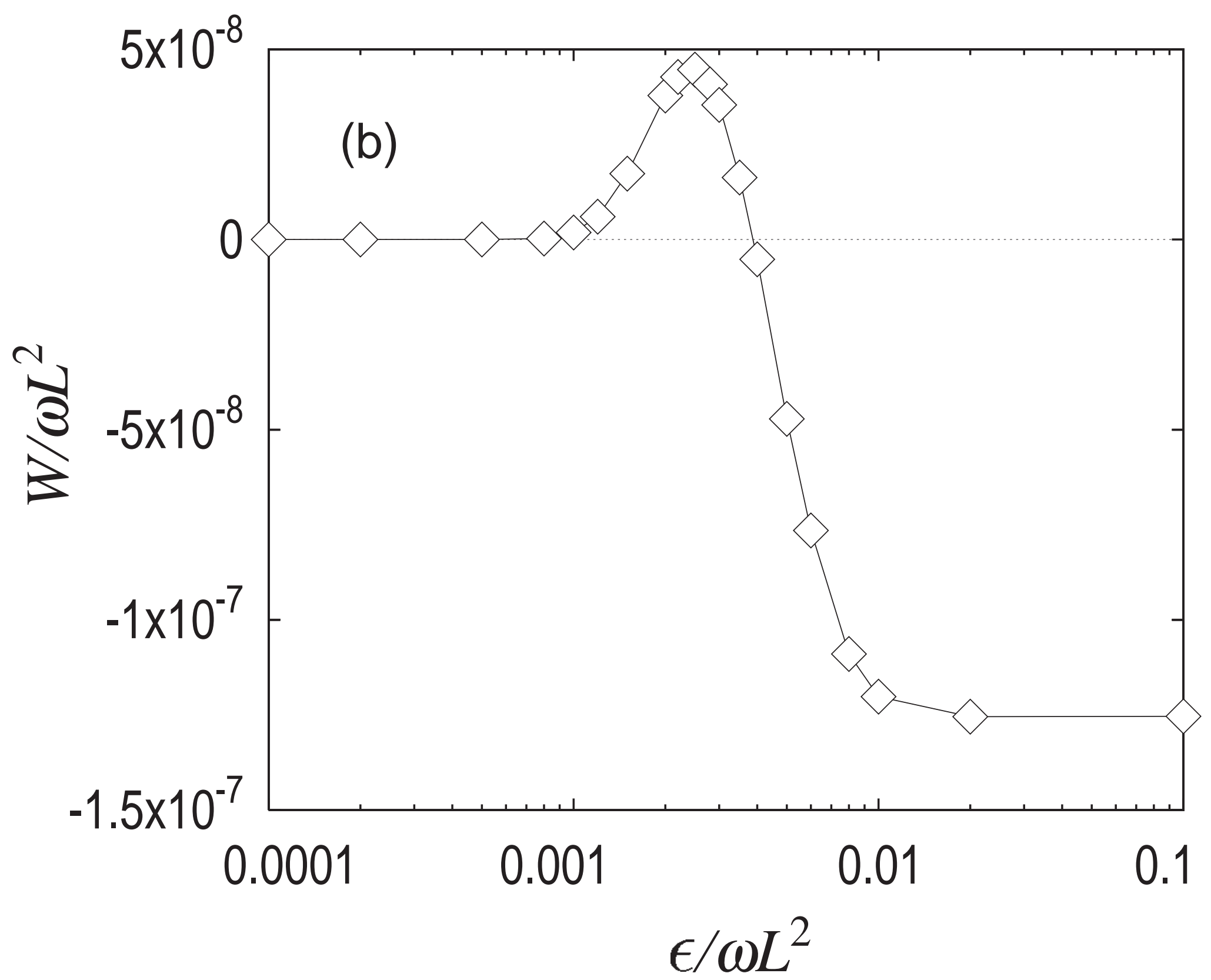




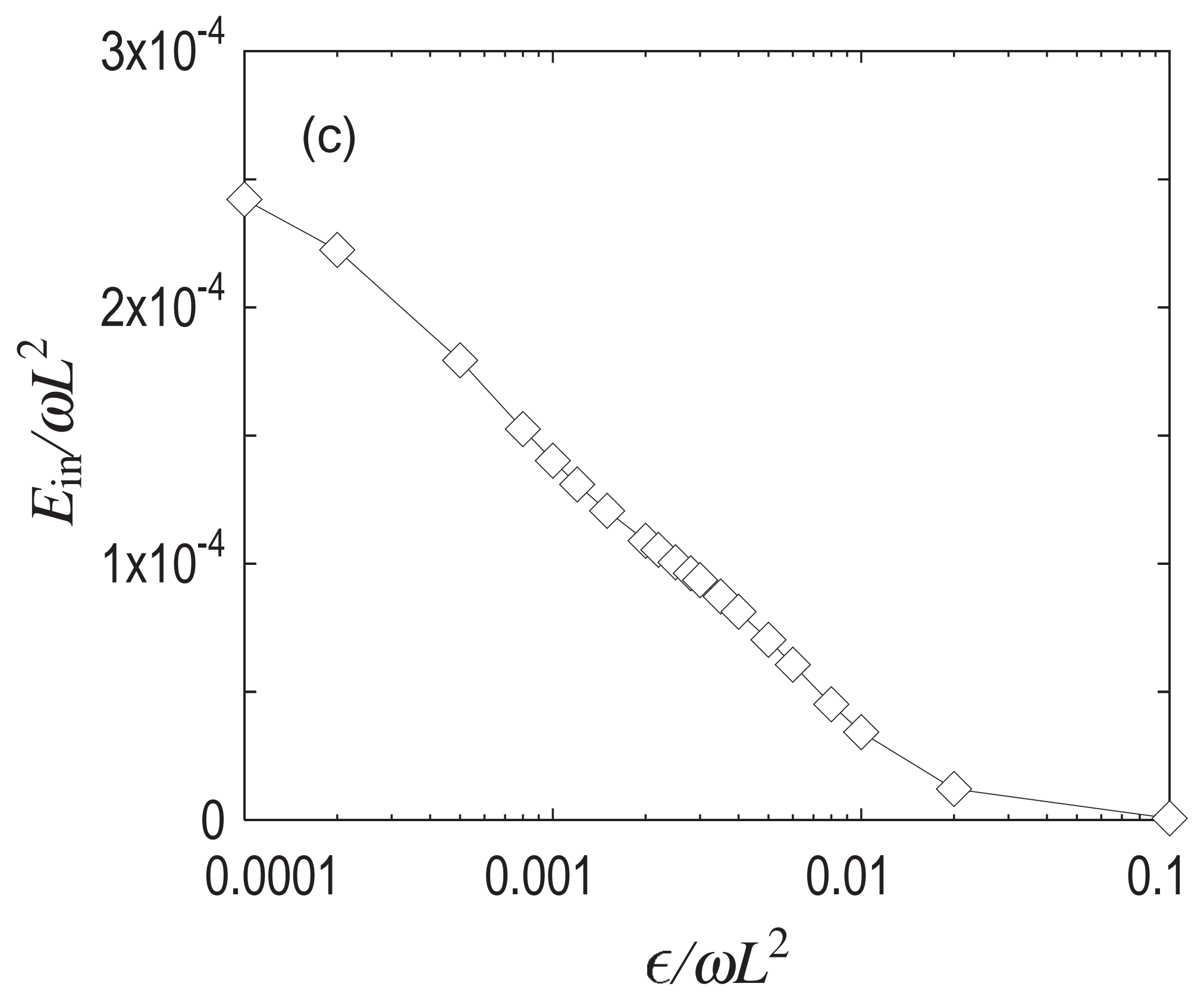




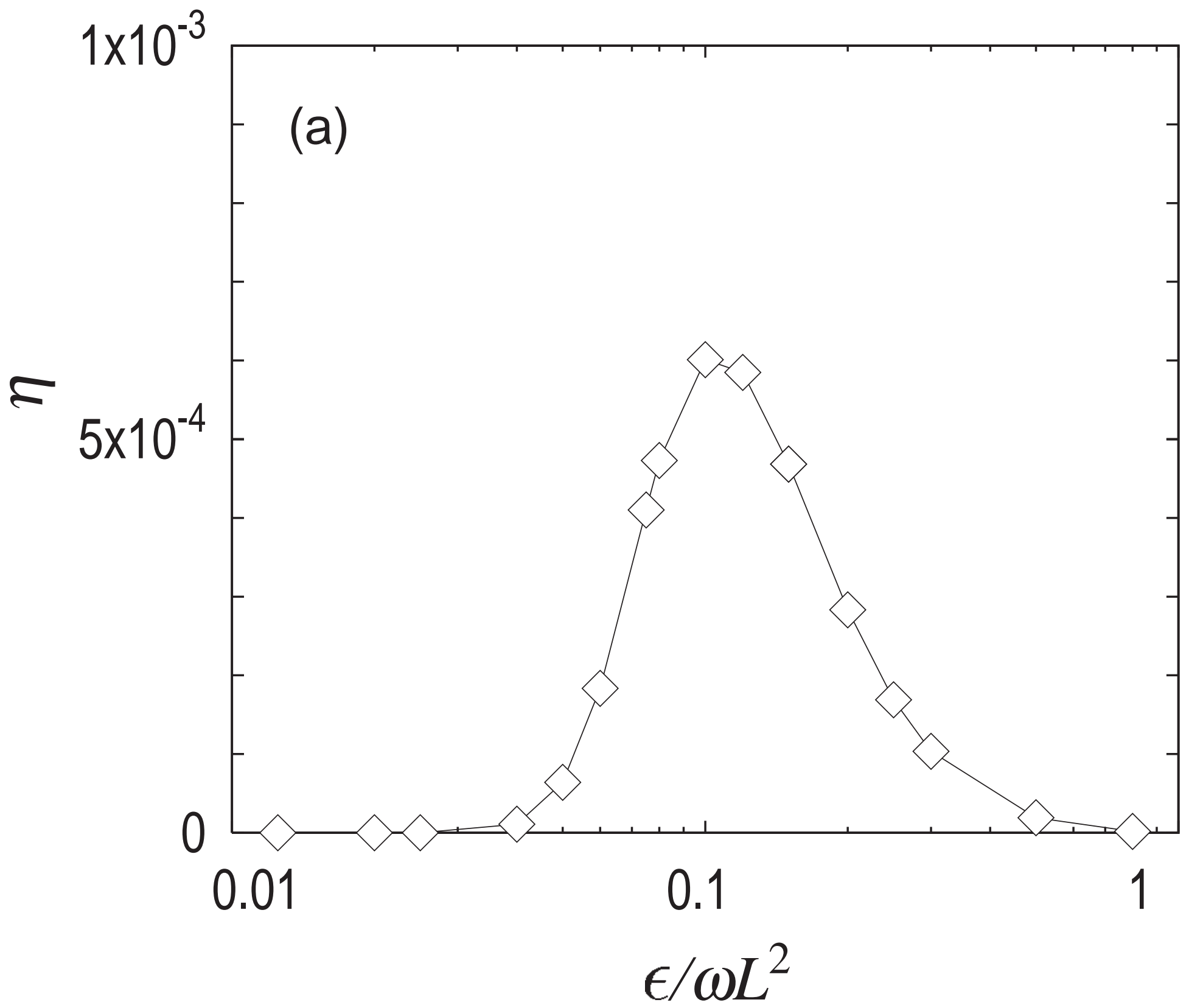




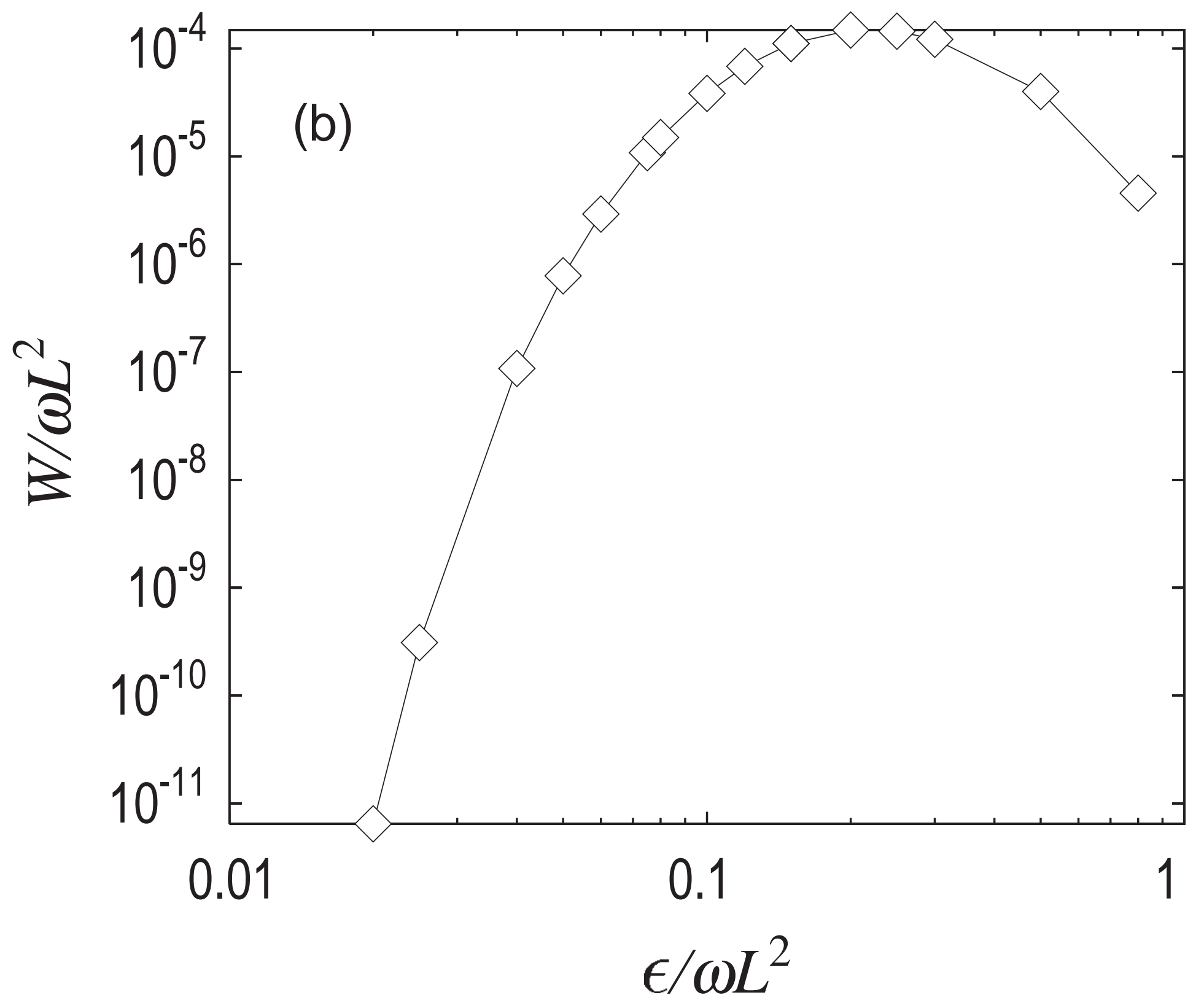




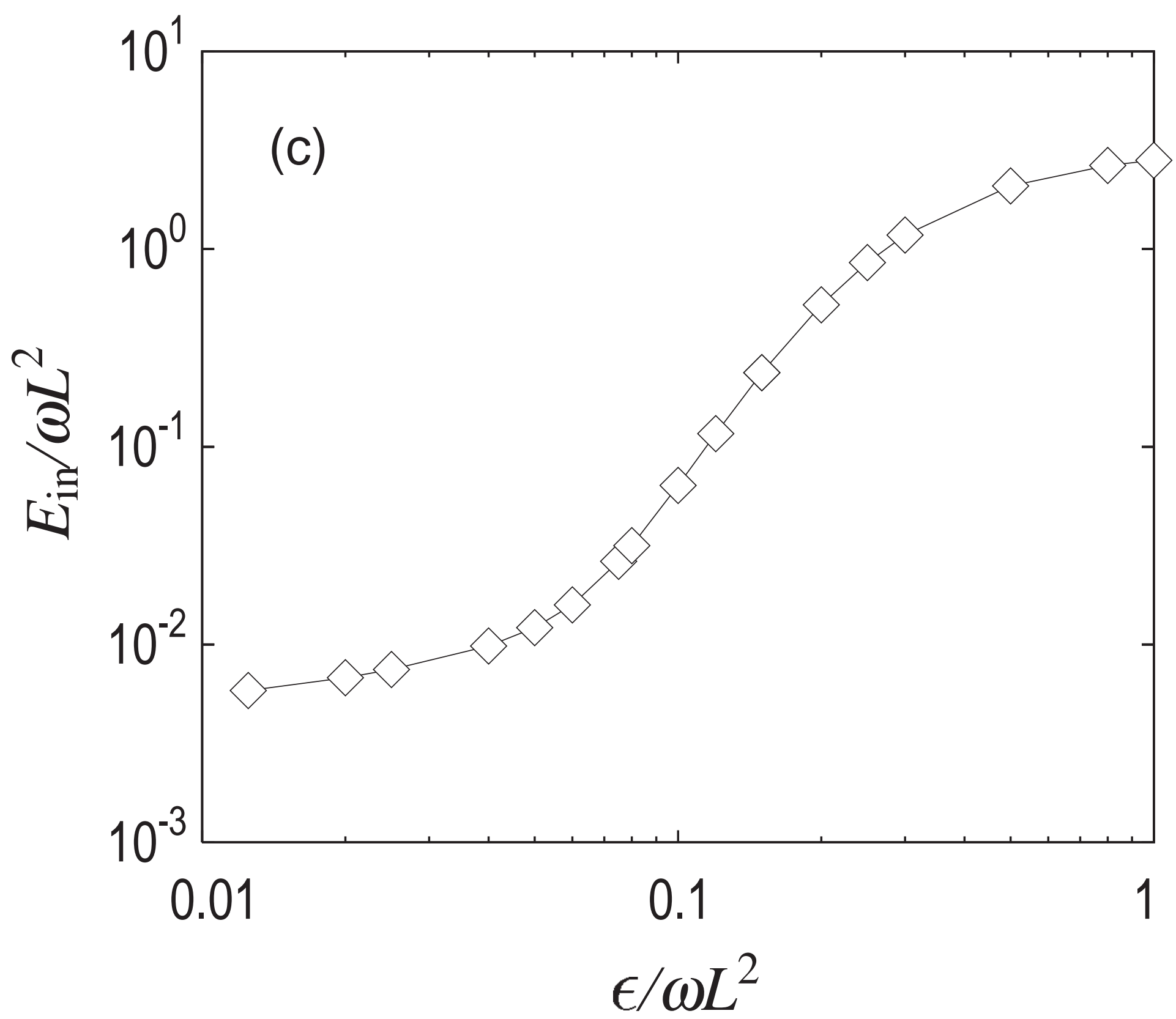

